Inertial sensing with point-source atom interferometry for interferograms with less than one fringe

Yun-Jhih Chen ${ }^{1,2}$, Azure Hansen ${ }^{1}$, Moshe Shuker ${ }^{1,3}$, Rodolphe Boudot ${ }^{1,4}$, John Kitching ${ }^{1}$, and Elizabeth A. Donley ${ }^{1}$

1. National Institute of Standards and Technology, Boulder, CO 80305 USA

2. University of Colorado, Boulder, CO 80309 USA

3. Rafael Ltd. (Israel)

4. FEMTO-ST, CNRS, 26 Rue de l'Epitaphe, 25030, Besançon, France

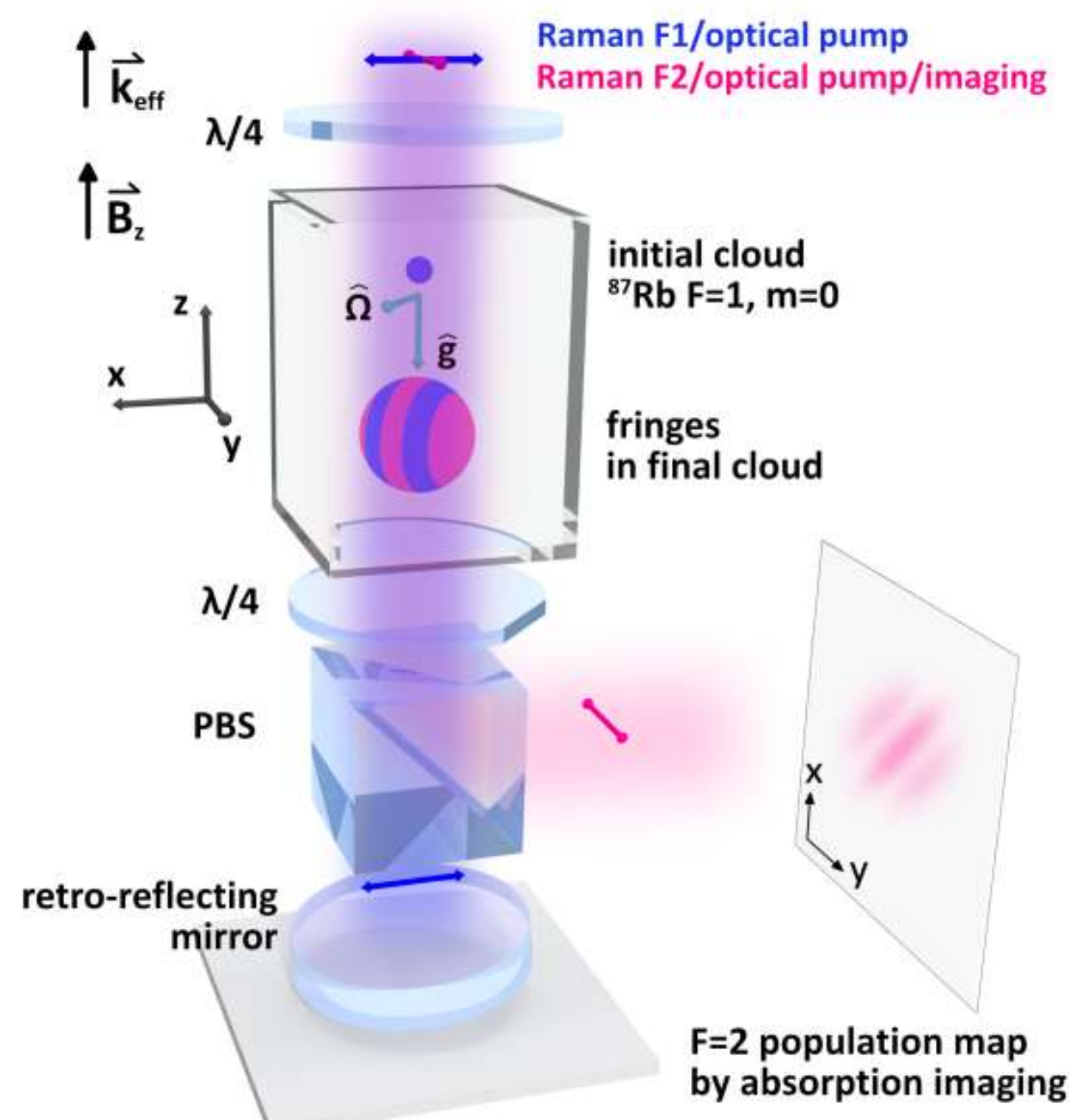




\section{Rotation measurement with interferometry}

"The Feynman path integral approach to atomic interferometry. A tutorial," Pippa Storey and Claude Cohen-Tannoudji, Journal de Physique II, EDP Sciences, 1994, 4 (11), pp.1999-2027.

\section{Sagnac phase shift}

$$
\begin{array}{rlr}
\delta \varphi_{\text {photon }} & =\frac{2 \omega_{0}}{c^{2}} A \Omega & \text { Light wave } \\
\delta \varphi_{\text {atom }} & =\frac{2 M}{\hbar} A \Omega & \text { Matter wave }
\end{array}
$$
A: Sagnac area
$\Omega$ : rotation rate
M: mass of atom
$\omega_{0}$ : angular frequency of light
c: speed of light
$\hbar$ : reduced Plank constant

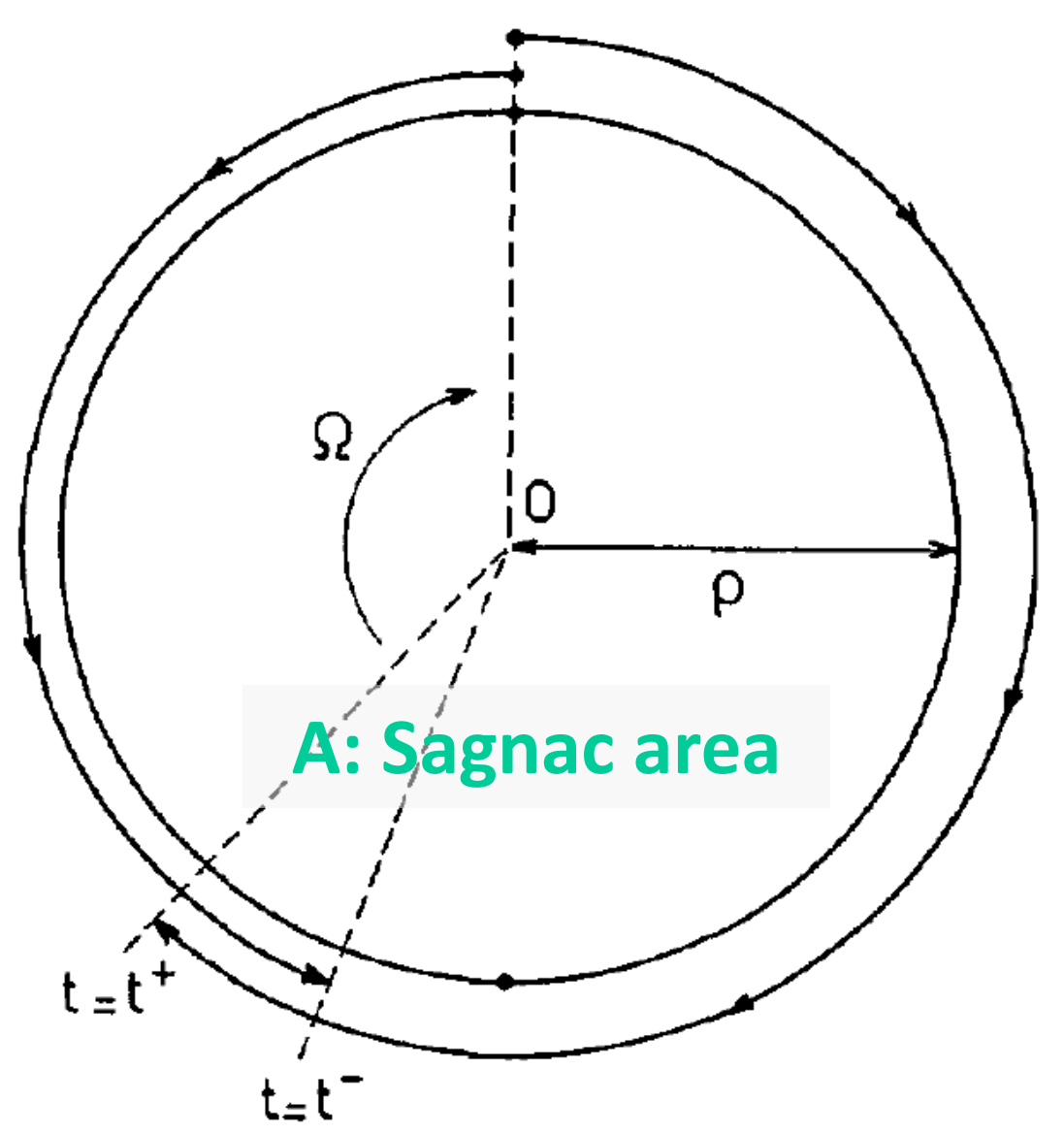




\section{Point source atom interferometry (PSI)}

1. PSI is a parallel operation of many different Sagnac interferometers.

2. PSI enables direct rotation measurement without ambiguity.

3. PSI resolves a rotation vector in a plane with high dynamic range.

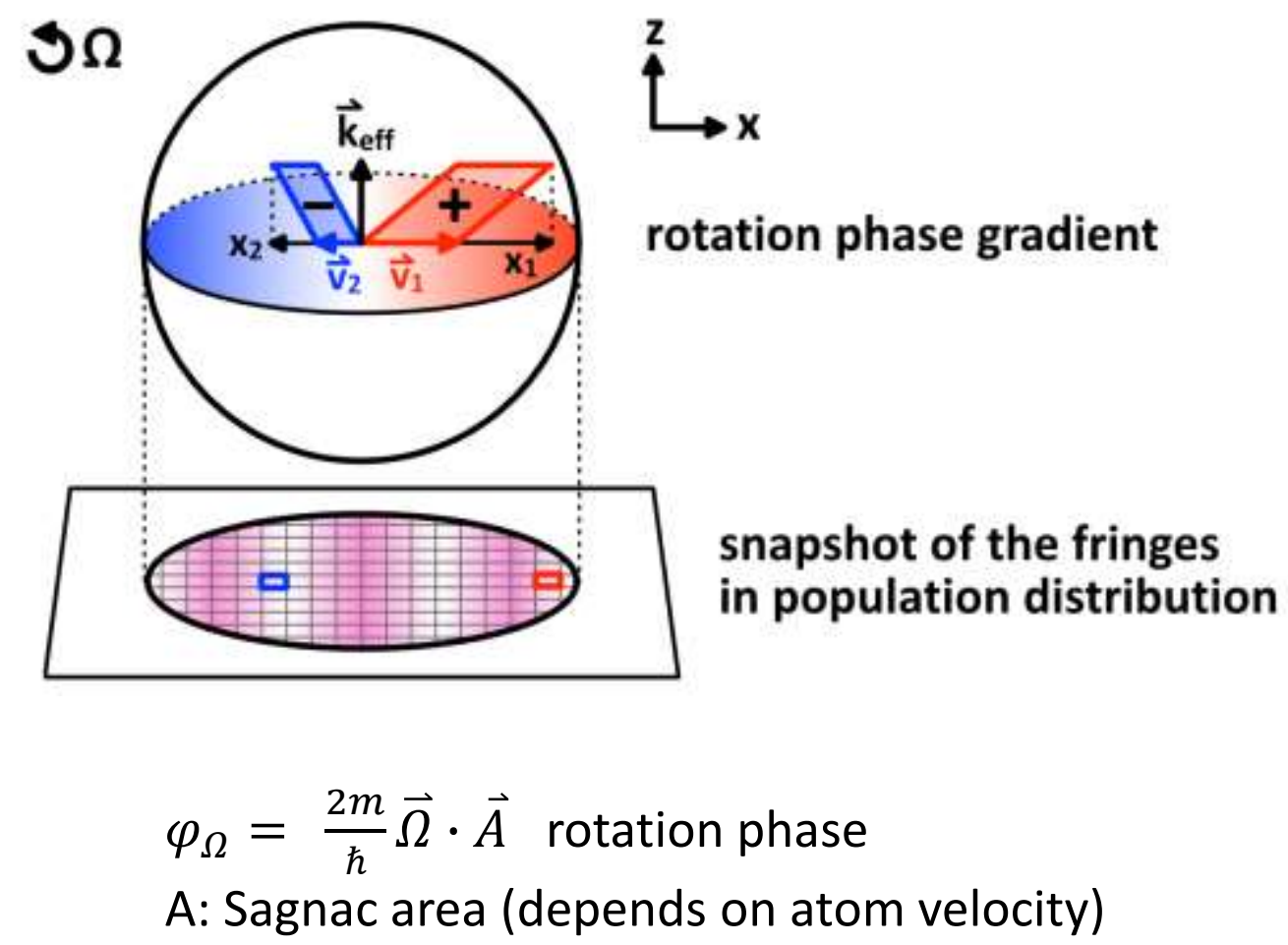




\section{Acknowledgement}

Rodolphe Boudot

Liz Donley

Azure Hansen

Greg Hoth

Eugene Ivanov

John Kitching

William McGehee

Bruno Pelle

Stefan Riedl

Moshe Shuker
PSI team reunion at IFCS-EFTF, April 2019

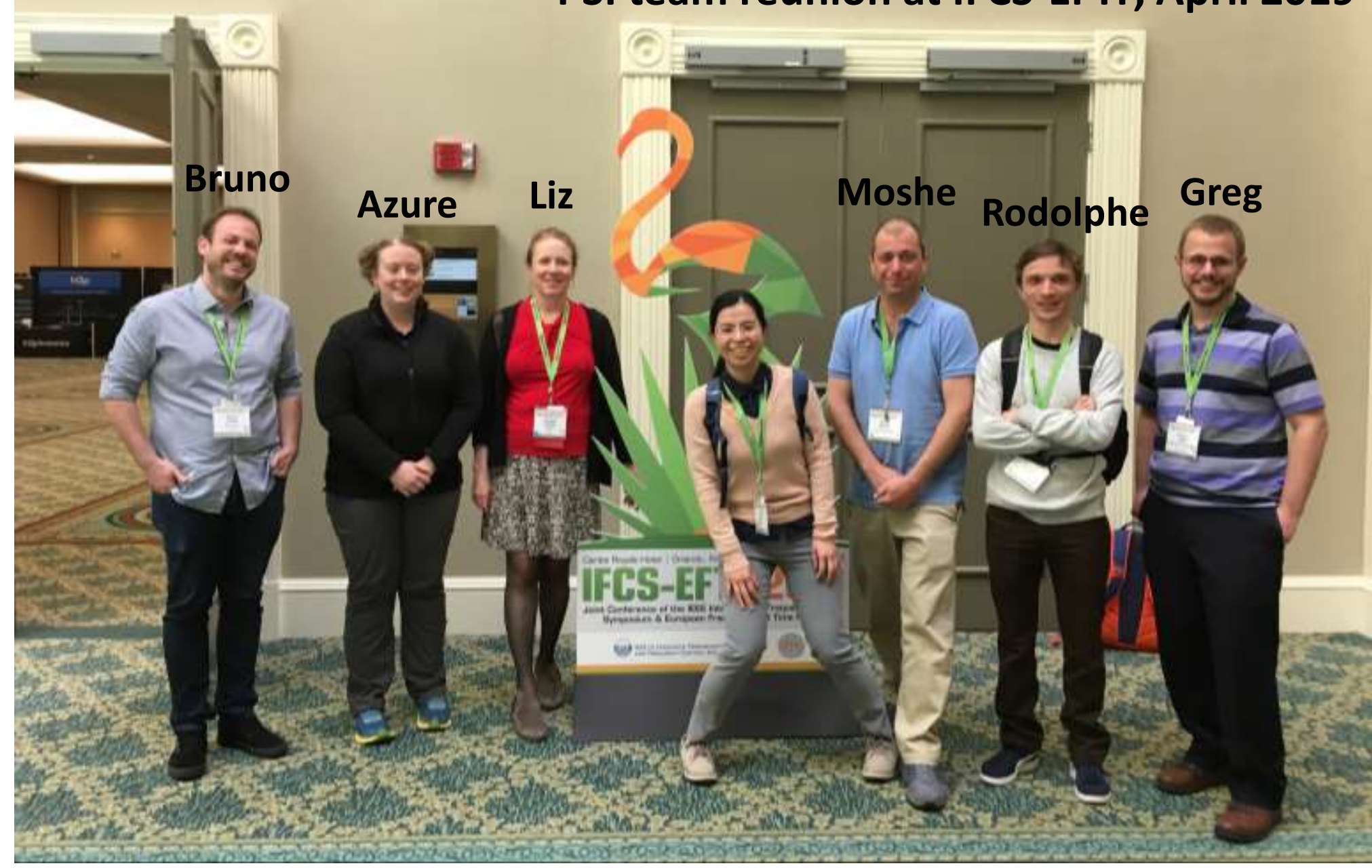




\section{Expanding point-like atomic source}

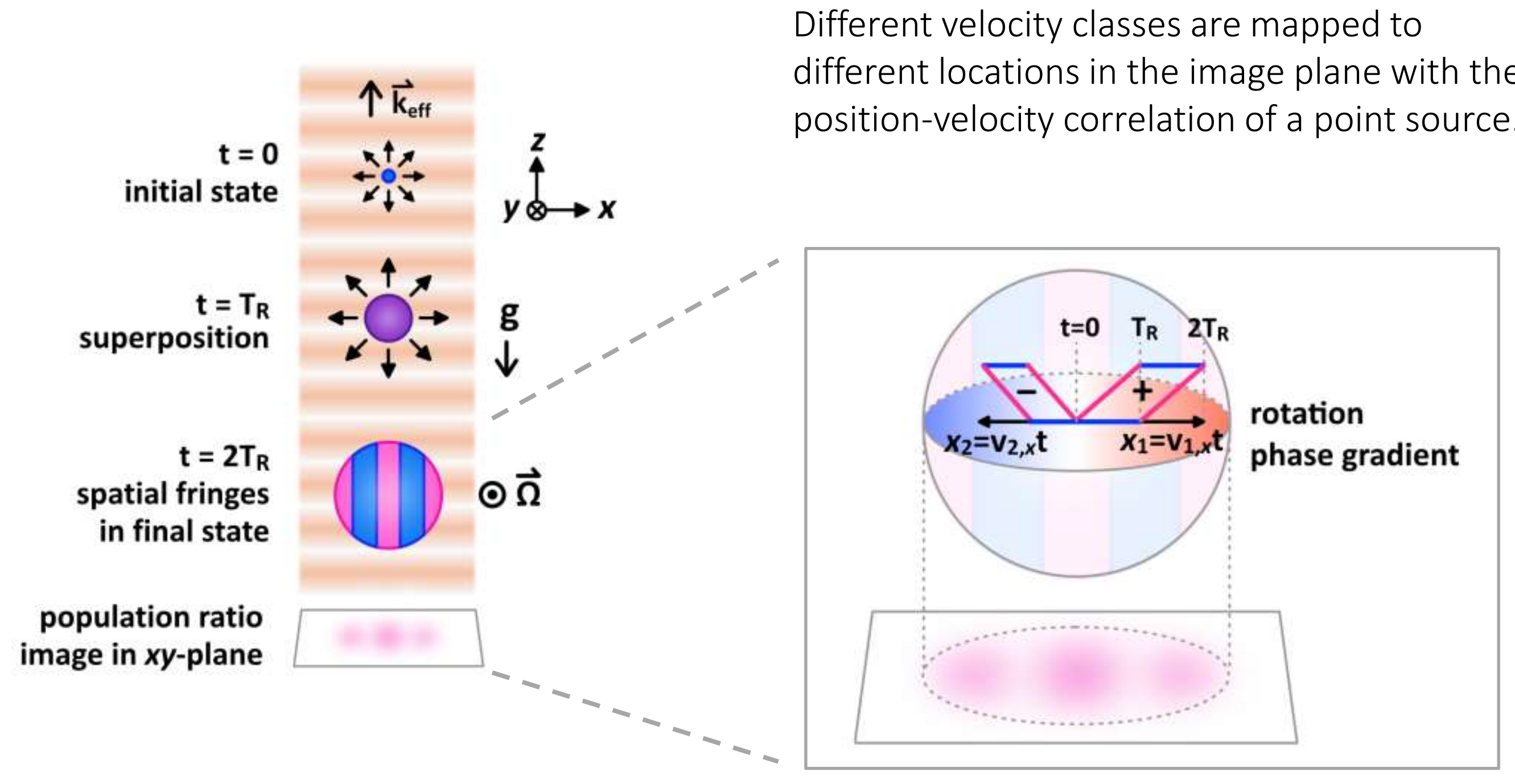




\section{Rotation phase gradient}

Interferometer phase shift of the

Raman $\pi / 2-\pi-\pi / 2$ pulse sequence:

$\varphi=\varphi_{a}+\varphi_{0}+\varphi_{\Omega}$

$\varphi_{a}=\vec{k}_{\text {eff }} \cdot \vec{a} T_{R}^{2}$ acceleration

$\varphi_{\Omega}=2 \vec{k}_{\text {eff }} \cdot(\vec{\Omega} \times \vec{v}) T_{R}^{2} \quad$ velocity

$\varphi_{0}=$ laser phase

dependent!

$T_{R}$ : time between Raman laser pulses
Position-velocity correlation of an ideal expanding point source: $\vec{v}=\frac{\vec{r}}{T_{\mathrm{ex}}}$

$T_{\mathrm{ex}}$ : cloud expansion time

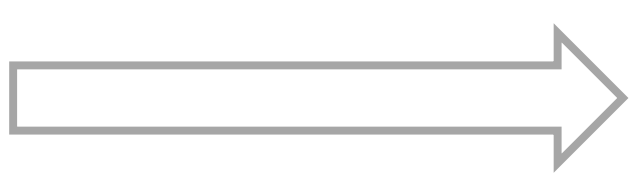

$\varphi_{\Omega}(\vec{r})=\vec{k}_{\Omega} \cdot \vec{r} \quad$ rotation

$\vec{k}_{\Omega}=\frac{2 T_{R}^{2}}{T_{\mathrm{ex}}} k_{\mathrm{eff}} \Omega \hat{n} \quad$ phase gradient

$\hat{n}=\hat{k}_{\text {eff }} \times \widehat{\Omega}$

$\varphi_{a}$ and $\varphi_{0}$ : constant across cloud
Fringe period : rotation rate

i Fringe direction : direction of the rotation vector

Fringe phase

: acceleration

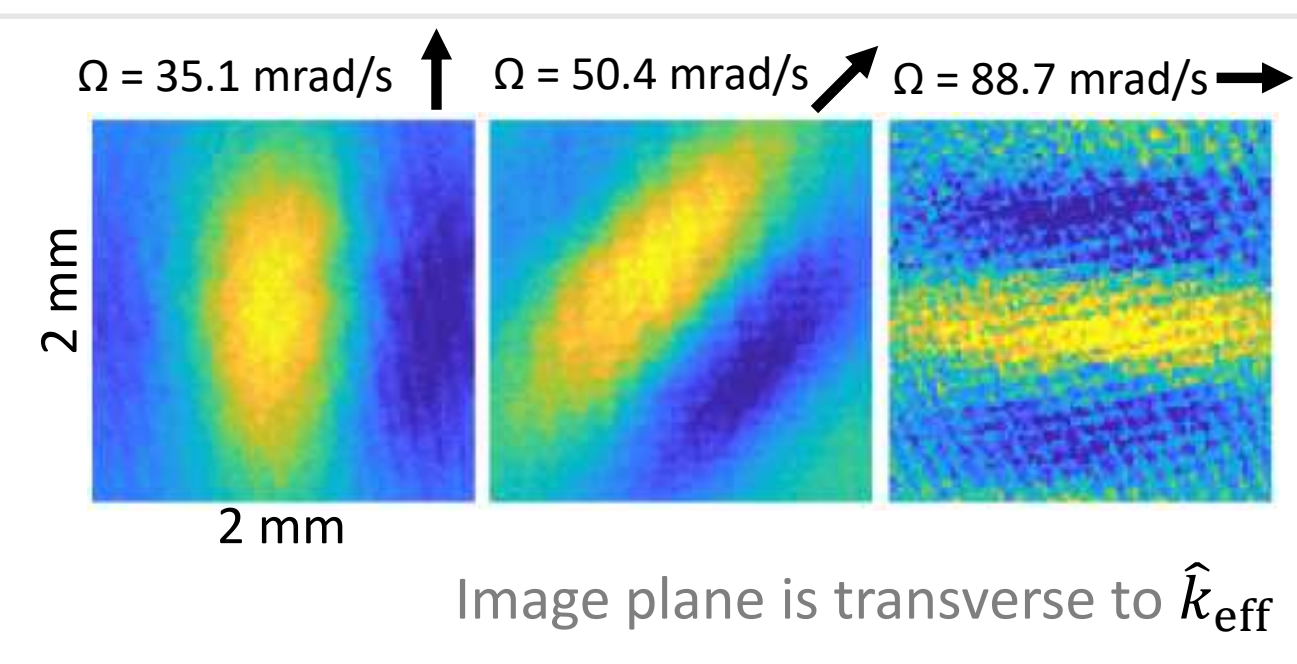




\section{Unambiguous rotation measurement}

Conventional:

Population ratio $\rightarrow$ phase $\rightarrow$ rotation

PSI:

Fringe period $\rightarrow$ phase gradient $\rightarrow$ rotation
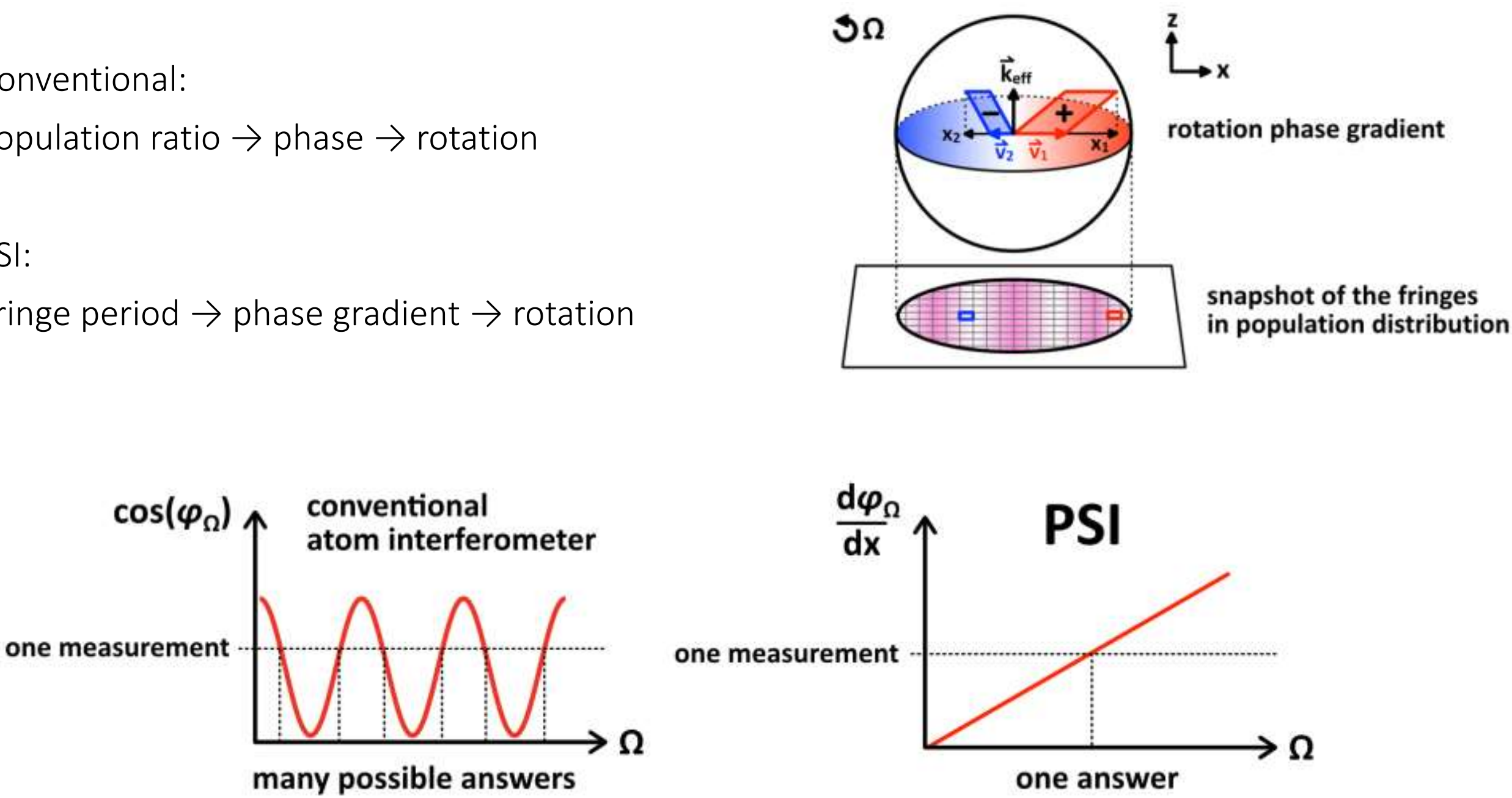


\section{PSI references}

Multiaxis inertial sensing with long-time point source atom interferometry

Enhanced Atom Interferometer Readout through the Application of Phase Shear

Point source atom interferometry with a cloud of finite size

Single-source multiaxis cold-atom interferometer in a centimeter-scale cell

Concept study and preliminary design of a cold atom interferometer for space gravity gradiometry

Rotation sensing with improved stability using pointsource atom interferometry

A Multi-Axis Atom Interferometer Gyroscope Based on a Grating Chip

High sensitivity multi-axes rotation sensing using large momentum transfer point source atom interferometry

Robust inertial sensing with point-source atom interferometry for interferograms spanning a partial period

\section{Authors}

Susannah M. Dickerson, Jason M. Hogan, Alex Sugarbaker, David M. S. Johnson, and Mark A. Kasevich

Alex Sugarbaker, Susannah M. Dickerson, Jason M. Hogan, David M. S. Johnson, and Mark A. Kasevich

Gregory W. Hoth, Bruno Pelle, Stefan Riedl, John Kitching, and Elizabeth A. Donley

Yun-Jhih Chen, Azure Hansen, Gregory W. Hoth, Eugene Ivanov, Bruno Pelle, John Kitching, and Elizabeth A. Donley

A. Trimeche, B. Battelier, D. Becker, A. Bertoldi, P. Bouyer, C. Braxmaier, E. Charron, R. Corgier, M. Cornelius, K. Douch, N. Gaaloul, S. Herrmann, J. Müller, E. Rasel, C. Schubert, H. Wu and F. Pereira dos Santos

Chen Avinadav, Dimitry Yankelev, Moshe Shuker, Ofer

Firstenberg, and Nir Davidson

Xiaojie Li, Zhixin Meng, Peiqiang Yan, Jianwei Zhang, Yanying Feng

Jinyang Li, Gregório R. M. da Silva, Wayne C. Huang, Mohamed Fouda, Timothy L. Kovachy, and Selim M. Shahriar

Yun-Jhih Chen, Azure Hansen, Moshe Shuker, Rodolphe Boudot, John Kitching, and Elizabeth A. Donley

\section{Link}

Year

https://doi.org/10.1103/ PhysRevLett.111.083001

https://doi.org/10.1103/ PhysRevLett.111.113002

https://doi.org/10.1063/ 1.4961527

https://doi.org/10.1103/ PhysRevApplied.12.0140 $\underline{19}$

https://doi.org/10.1088/ 1361-6382/ab4548

https://doi.org/10.1103/ PhysRevA.102.013326

https://doi.org/10.1109// NERTIAL48129.2020.909 $\underline{0092}$

https://arxiv.org/abs/200 $\underline{6.13442}$

https://doi.org/10.1364/ OE.399988 


\section{NIST ADI group's PSI gyro}

Experimental scheme:

1. MOT, compressed MOT, and molasses

2. State preparation

3. Raman interrogations

4. State-selective absorption imaging

Repetition rate: 5 to $10 \mathrm{~Hz}$

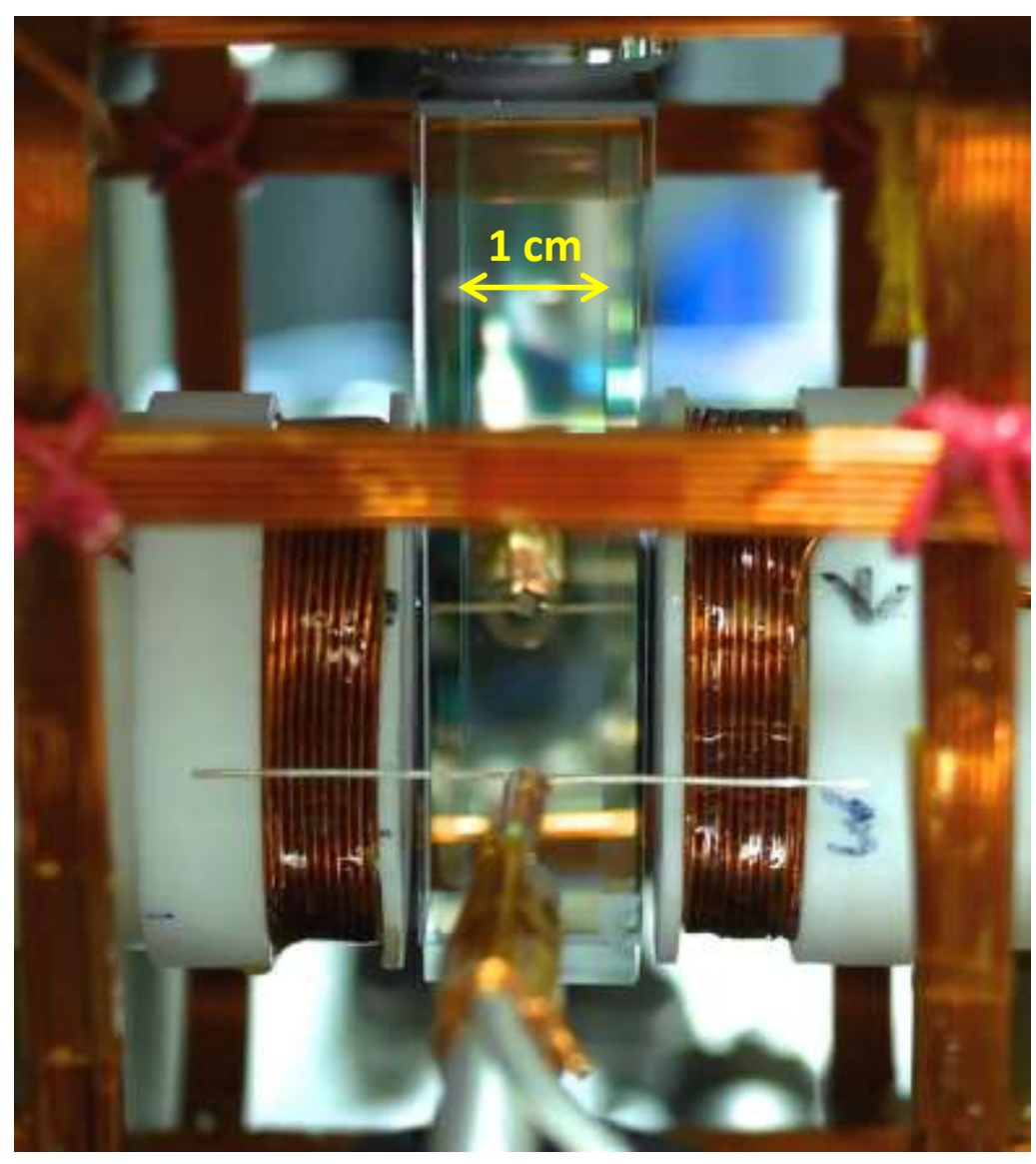



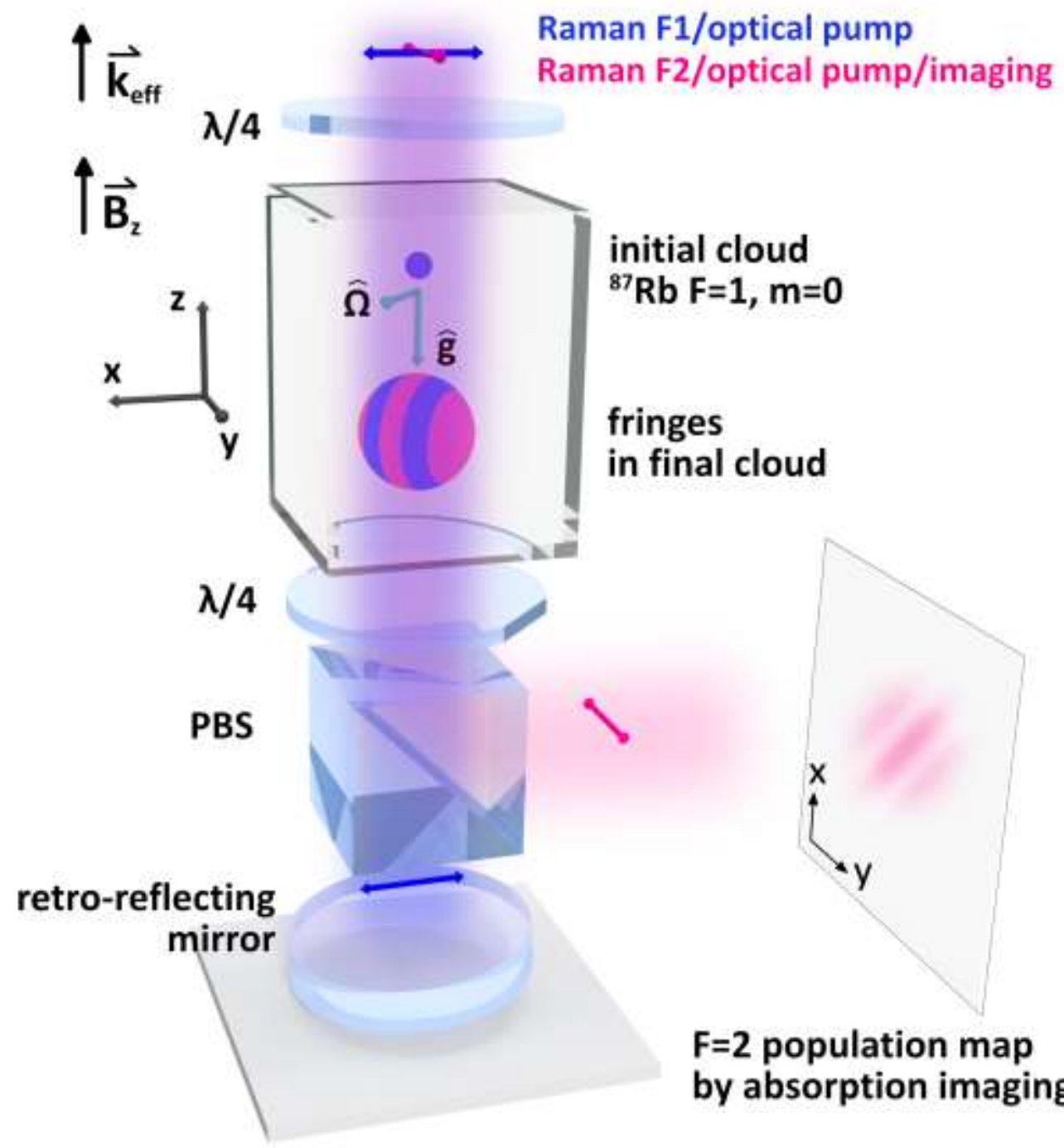

initial cloud

${ }^{87} \mathrm{Rb} F=1, m=0$

fringes

in final cloud

$\mathrm{F}=2$ population map by absorption imaging

\section{two-photon Raman transition}
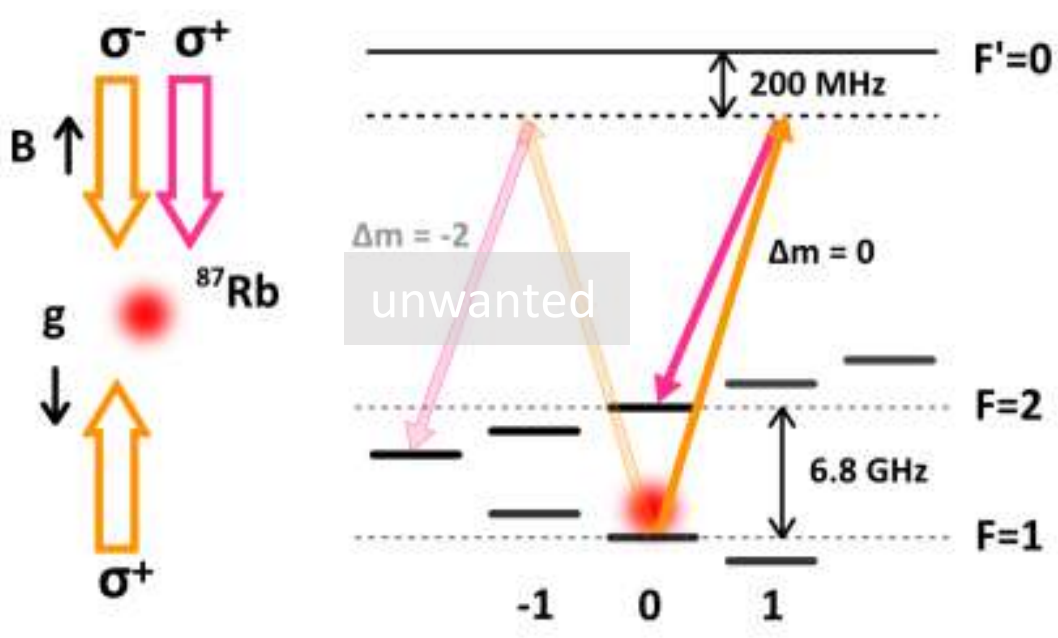


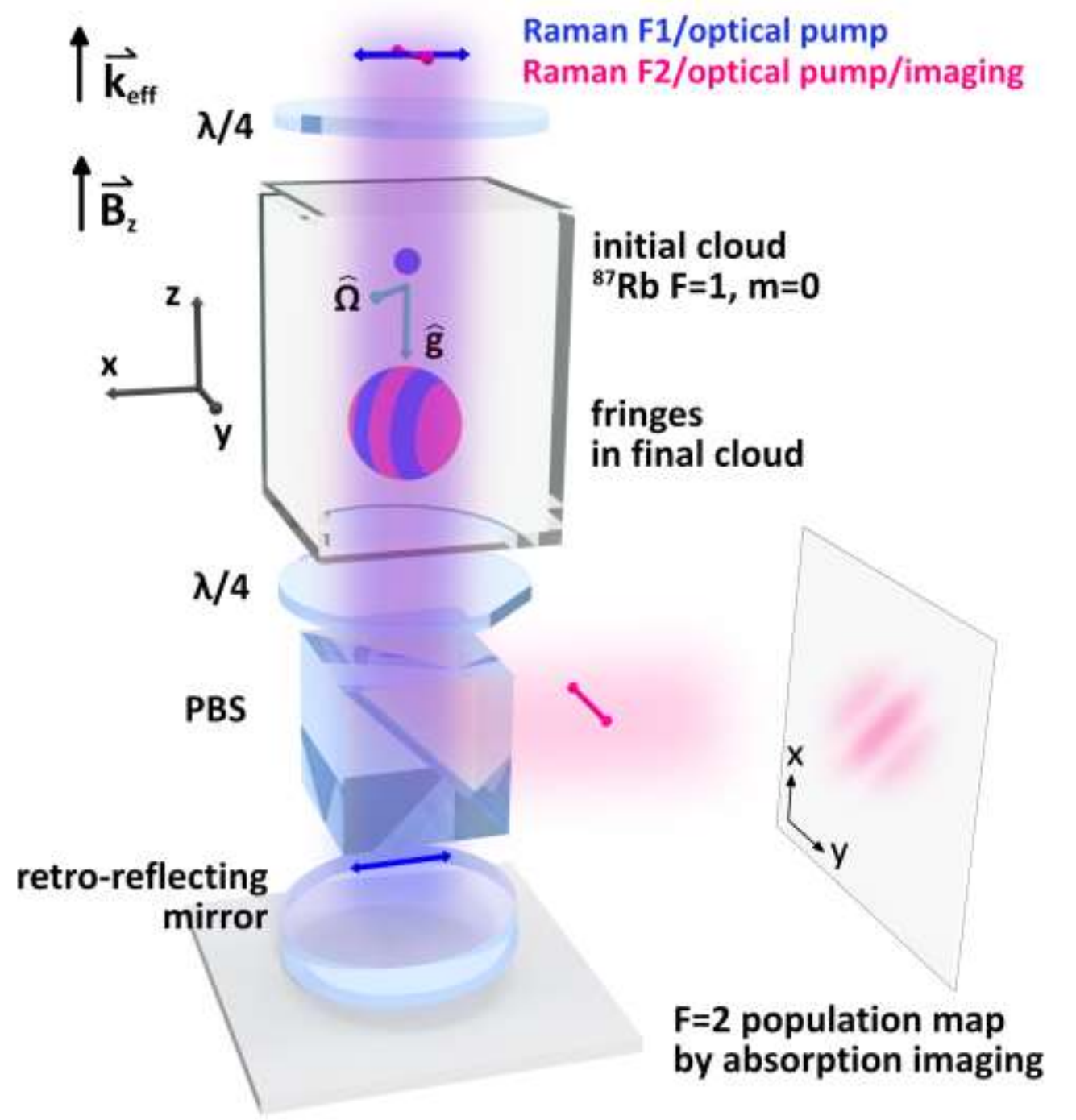

\section{Multi-axis sensitivity}

The effective wave vector $k_{\text {eff }}$ is in $+z$ direction. Cloud is imaged in xy-plane. PSI measures:

1. Acceleration in the z-axis

2. Rotation projected onto the $x y$-plane

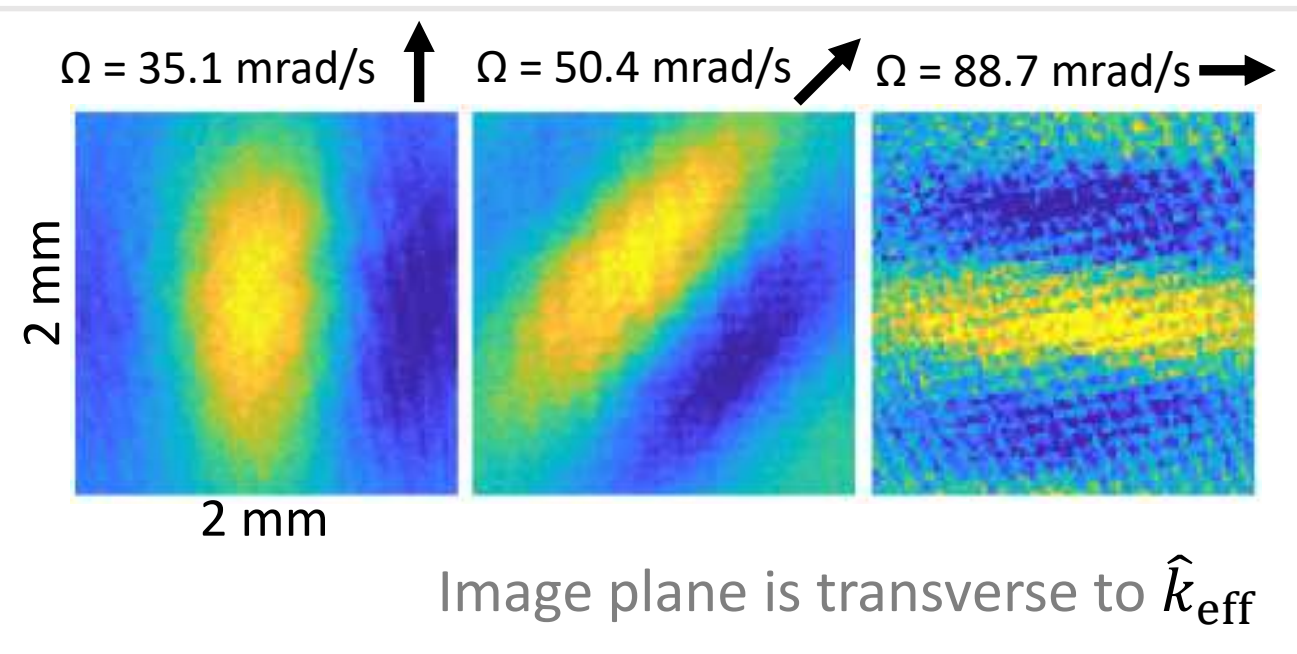




\section{Experimental demonstration of the two-dimensional rotation measurement with point source atom interferometry}

0 degrees
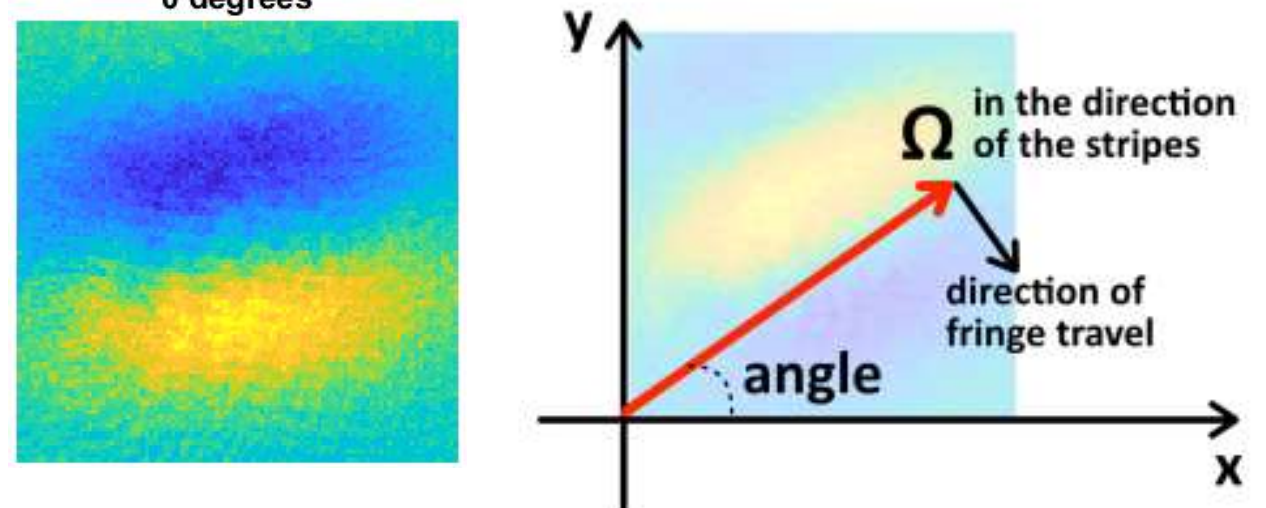

Fringe period : rotation rate

Fringe direction : direction of the rotation vector

Fringe phase acceleration

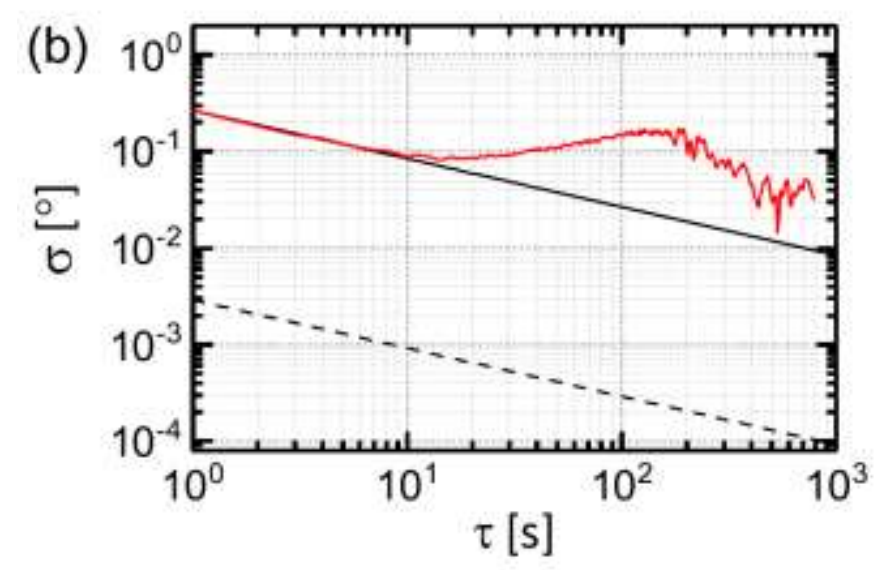

Direction of the rotation vector in a plane, $0.27^{\circ}$ at $\tau=1 \mathrm{~s}$

doi.org/10.1103/PhysRevApplied.12.014019

Application ideas:

1. navigation, gyrocompassing

2. Fundamental physics, relativistic precession measurement 
Measurement of small rotation rates
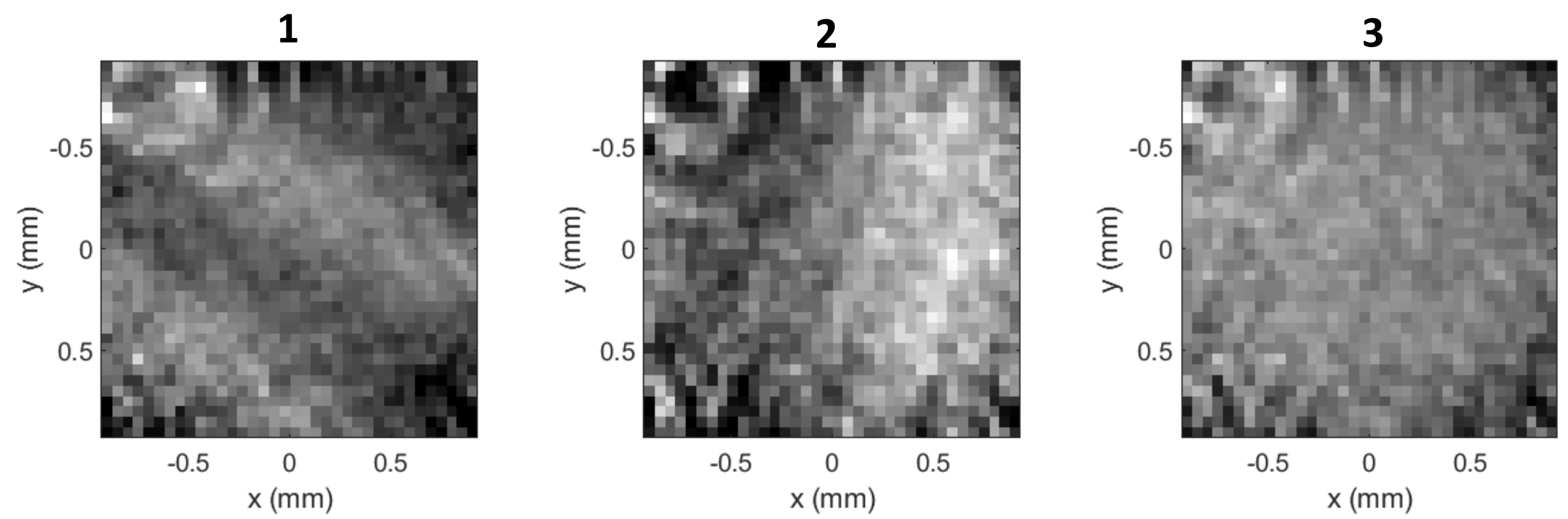

How to measure rotation in case 3 ? 


\section{Method in literature: phase shear}

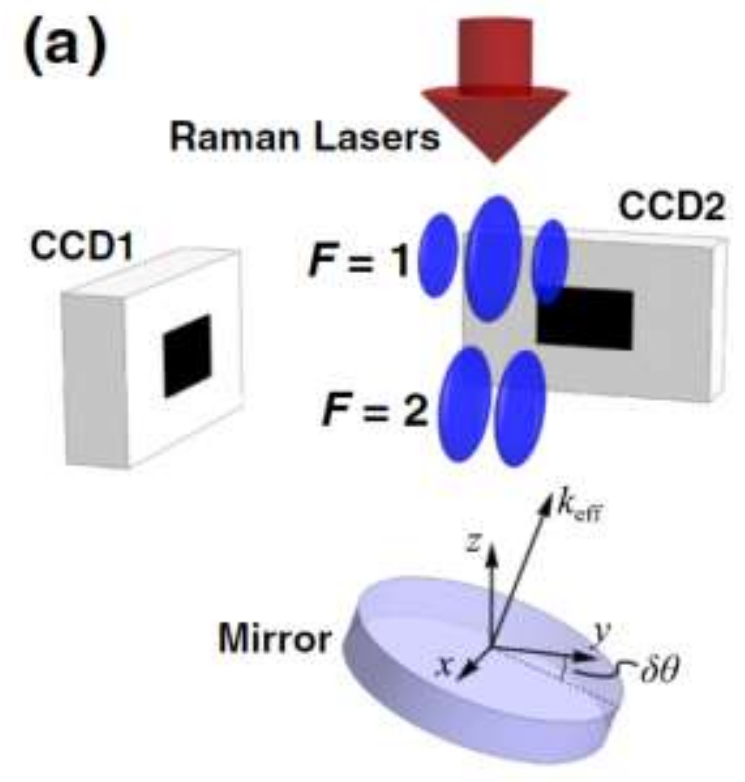

"Enhanced Atom Interferometer Readout through the Application of Phase Shear," A. Sugarbaker, S. M. Dickerson, J. M. Hogan, D. M. S. Johnson, and M. A. Kasevich, PRL 111, 113002 (2013).
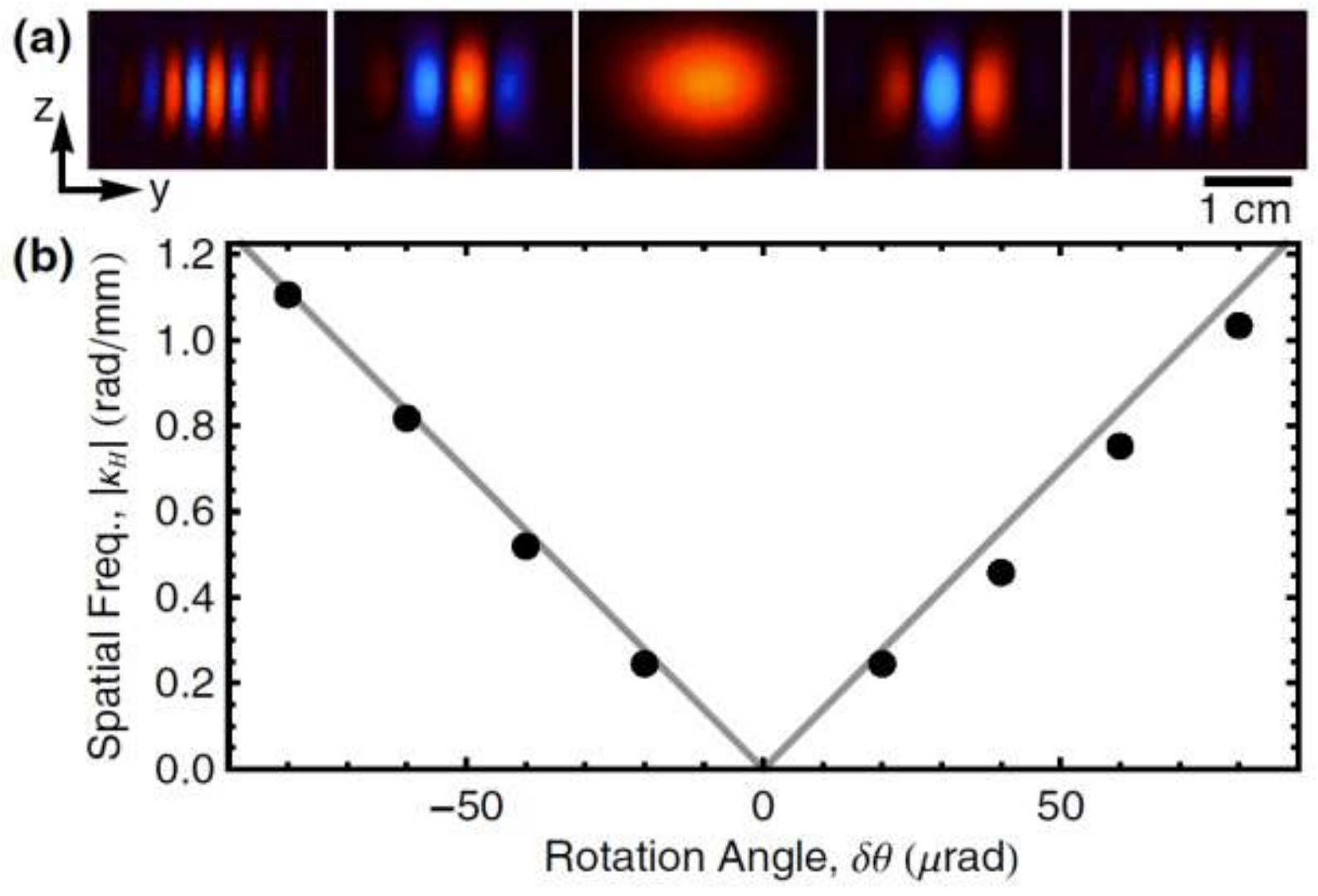


\section{Method in literature: ellipse fitting}

Ellipse equation:

$A x^{2}+B x y+C y^{2}+D x+E y+F=0$

Phase difference:

$\Delta \Phi_{L R}=\cos ^{-1}(-B / 2 \sqrt{A C})$

Left population ratio $=x \quad \mid$ Right population ratio $=y$

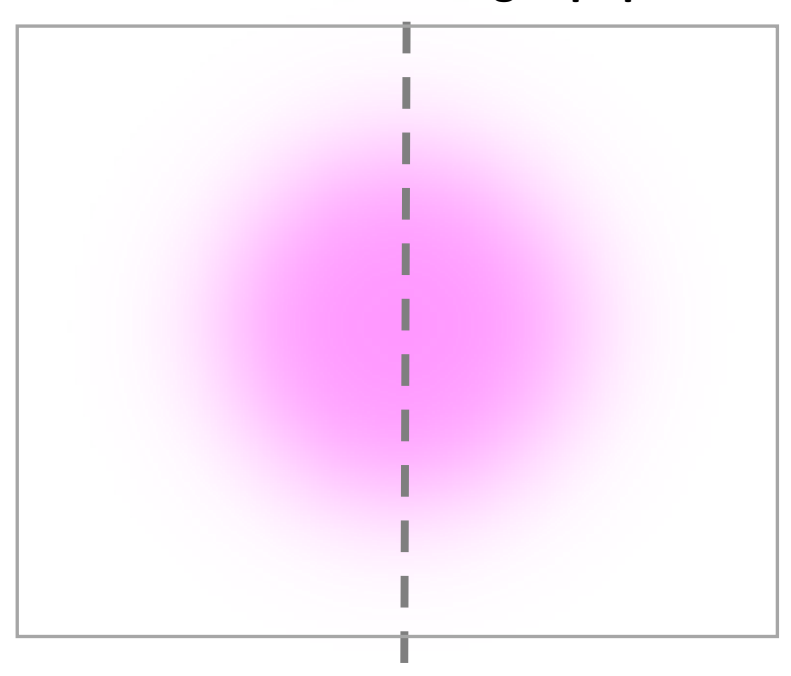

(a)

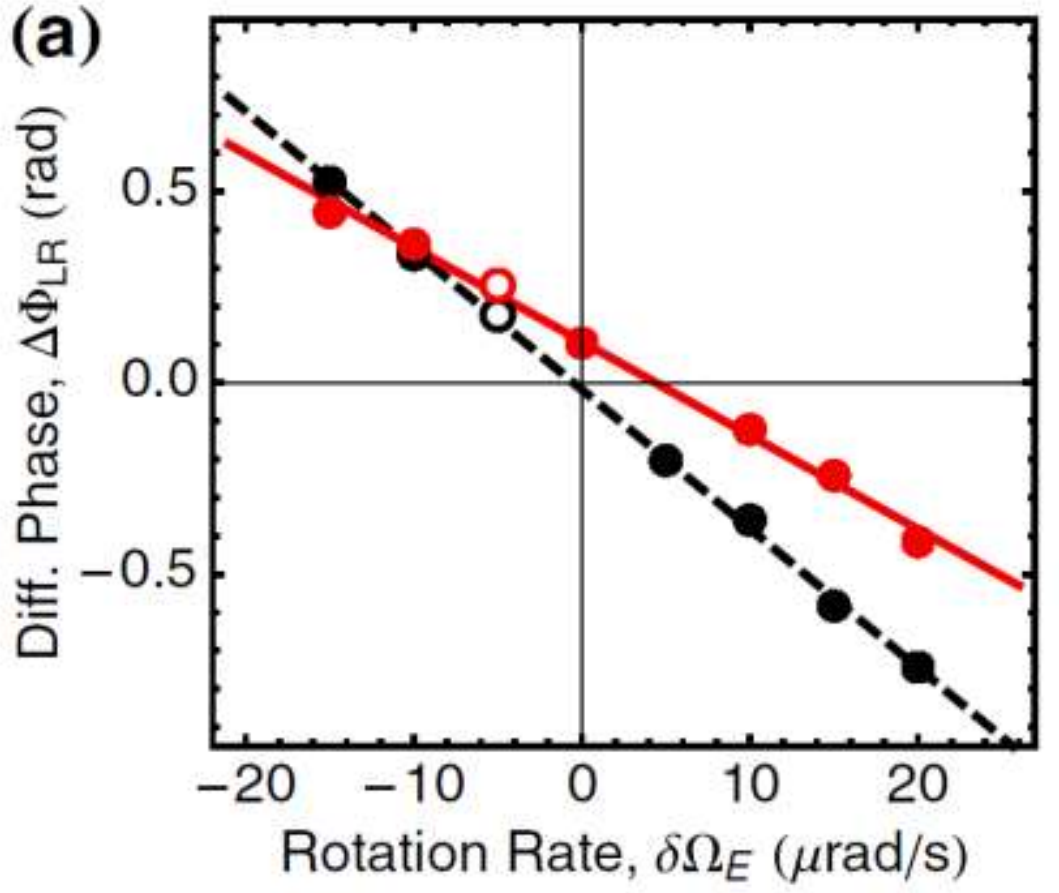

(b)

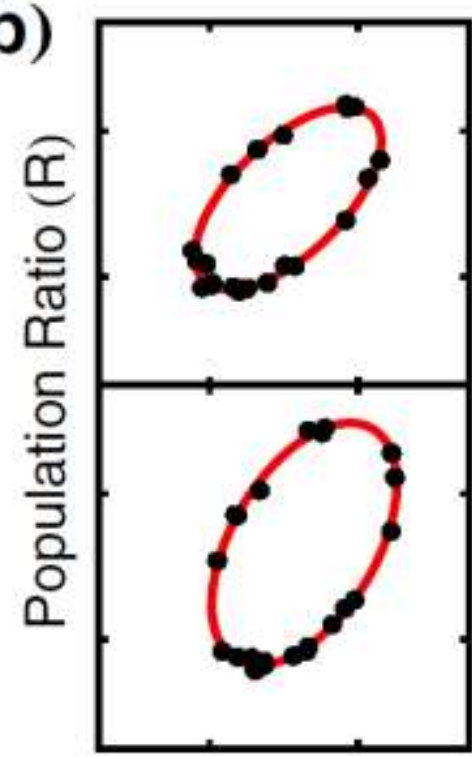

Pop. Ratio (L)

"Multiaxis Inertial Sensing with Long-Time Point Source Atom Interferometry," S. M. Dickerson, J. M. Hogan, A. Sugarbaker, D. M. S. Johnson, M. A. Kasevich, PRL 111, 083001 (2013). 


\section{Our approach}

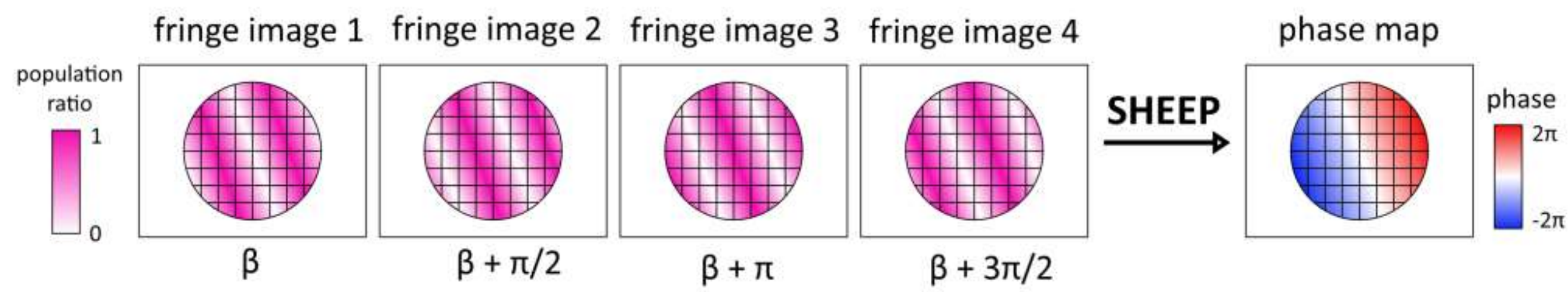

SHEEP-Simple, High dynamic range, and Efficient Extraction of Phase map

\section{https://doi.org/10.1364/OE.399988}




\section{Phase shifting interferometry}

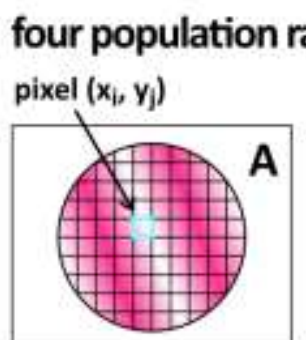

$\alpha_{0}$

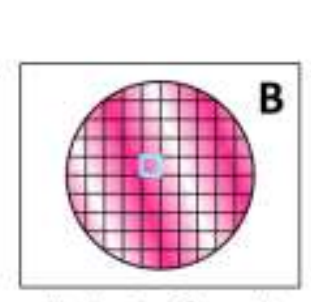

$(\Delta \alpha) \times 2 \pi T^{2}=\pi / 2$

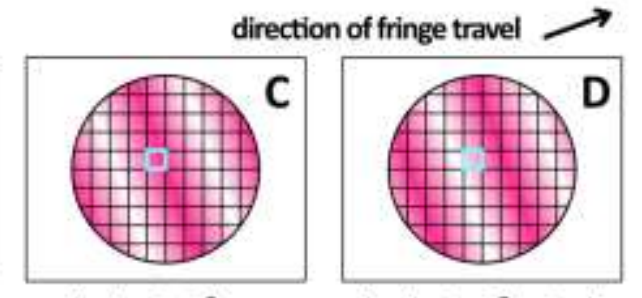

$(\Delta \alpha) \times 2 \pi T^{2}=3 \pi / 2$

$(\Delta \alpha) \times 2 \pi T^{2}=\pi$ population ratio of pixel $\left(x_{i}, y_{j}\right)$

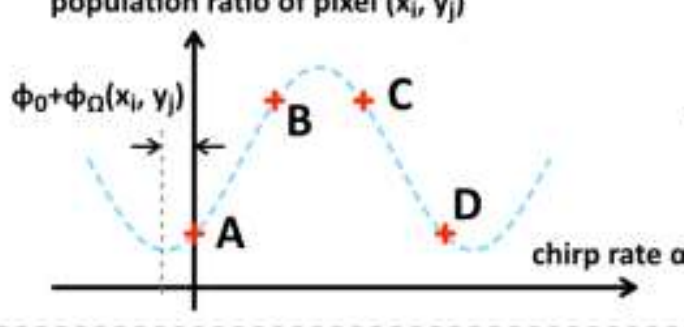

phase map $\phi_{0}+\phi_{\Omega}(x, y)$

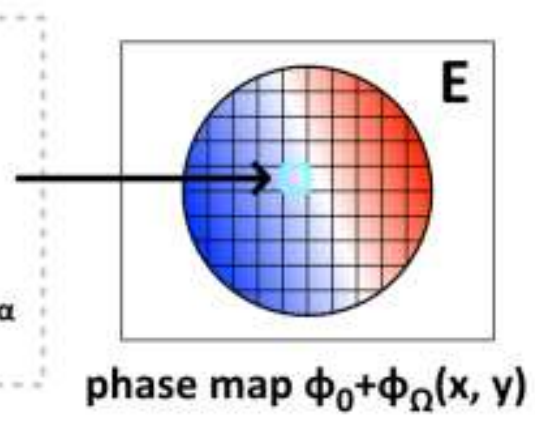

INPUT

Population ratio maps:

$A\left(x_{i}, y_{j}\right)=\frac{1}{2}\left\{1-c \cos \left[\varphi_{0}+\varphi_{\Omega}\left(x_{i}, y_{j}\right)\right]\right\}$

$B\left(x_{i}, y_{j}\right)=\frac{1}{2}\left\{1+c \sin \left[\varphi_{0}+\varphi_{\Omega}\left(x_{i}, y_{j}\right)\right]\right\}$

$C\left(x_{i}, y_{j}\right)=\frac{1}{2}\left\{1+c \cos \left[\varphi_{0}+\varphi_{\Omega}\left(x_{i}, y_{j}\right)\right]\right\}$

$D\left(x_{i}, y_{j}\right)=\frac{1}{2}\left\{1-c \sin \left[\varphi_{0}+\varphi_{\Omega}\left(x_{i}, y_{j}\right)\right]\right\}$

\section{OUTPUT}

\section{Phase map}

$$
\begin{aligned}
E\left(x_{i}, y_{j}\right) & =\tan ^{-1} \frac{B\left(x_{i}, y_{j}\right)-D\left(x_{i}, y_{j}\right)}{C\left(x_{i}, y_{j}\right)-A\left(x_{i}, y_{j}\right)}+m \pi \\
& =\varphi_{0}+\varphi_{\Omega}\left(x_{i}, y_{j}\right) \quad \text { Stitching the }
\end{aligned}
$$

\footnotetext{
Rotation phase gradient

: average of the differences in pixel values of adjacent pixels

i Acceleration phase

: average of the entire phase map
} 


\section{Case 3 revisited with SHEEP method}

1. The direction of the rotation traces a $360^{\circ}$ range in $2^{\circ}$ steps.

2. X-and y-components of the rotation vector vary sinusoidally.
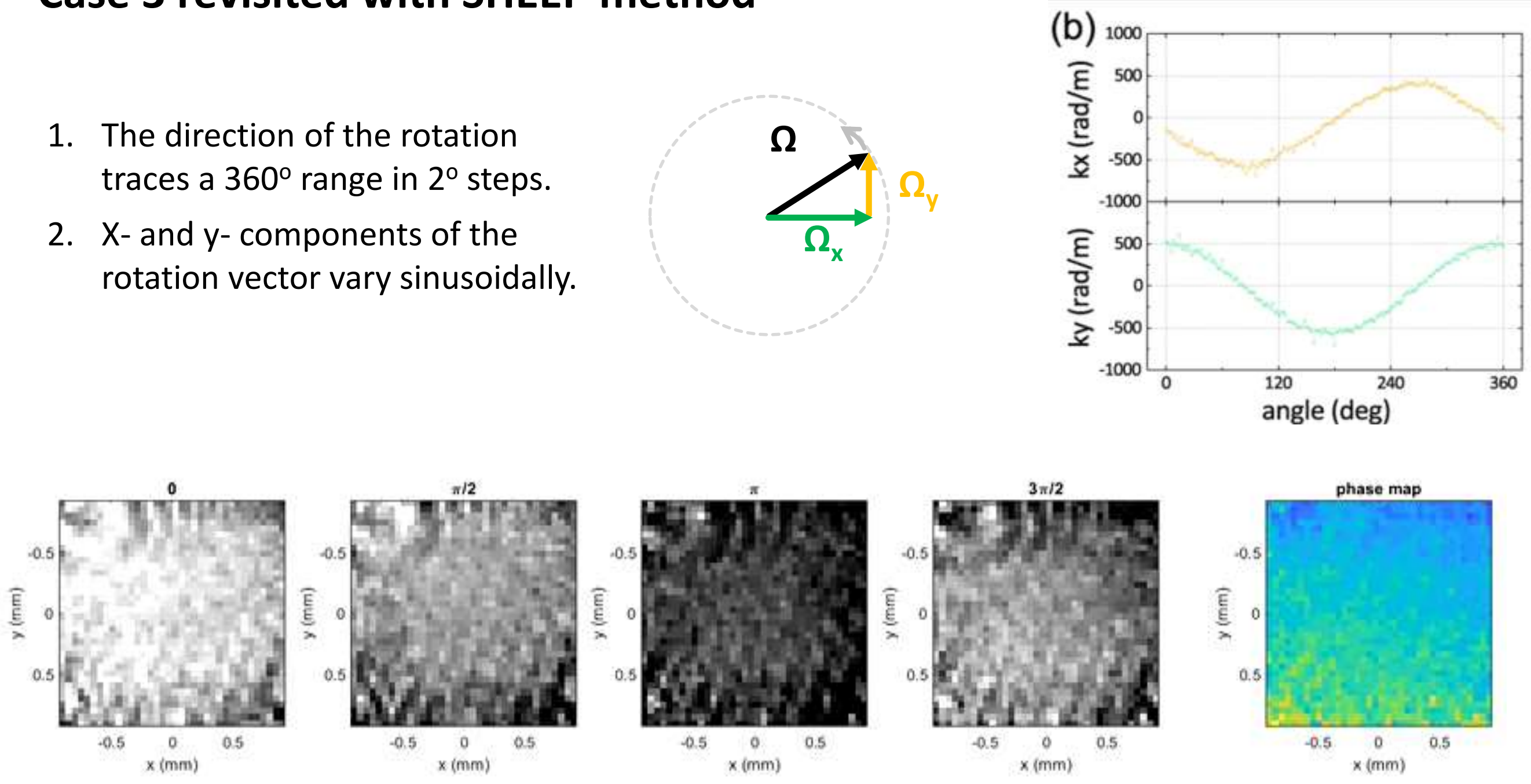


\section{Dynamic range}

- Robust and accurate

- Performs well over a wide range of rotation rates

- Lowest rotation rate $0.045^{\circ} / \mathrm{s}$ at T $=7.8 \mathrm{~ms}$, corresponding to 0.025 fringes in the image.

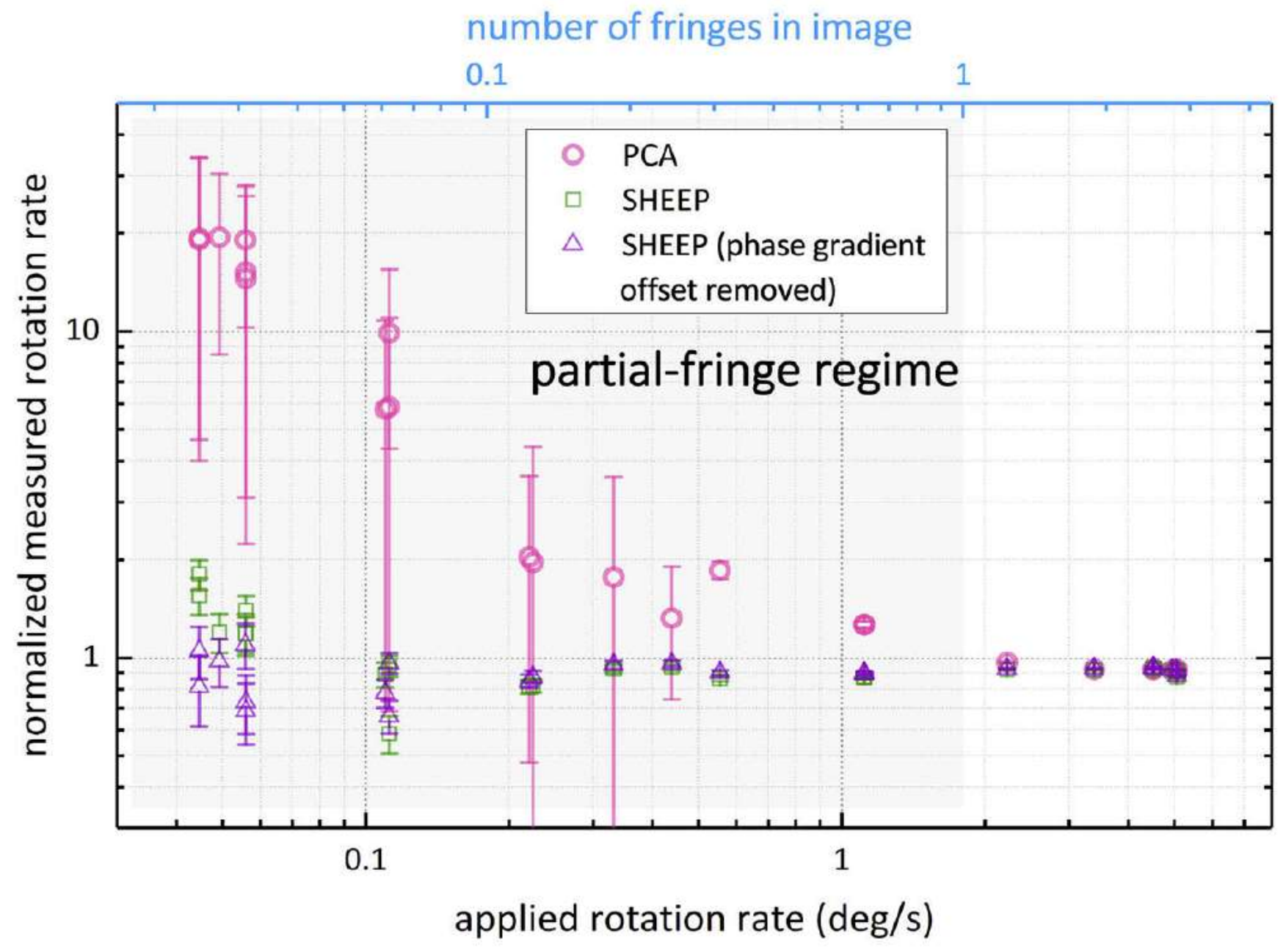




\section{Discussion}

\section{Vibrations}

- Spurious phase between images

- Sensitivities:

$$
\begin{array}{ll}
\text { Acceleration } & \propto T^{2} \\
\text { Rotation } & \propto T
\end{array}
$$

- Closed-loop operation

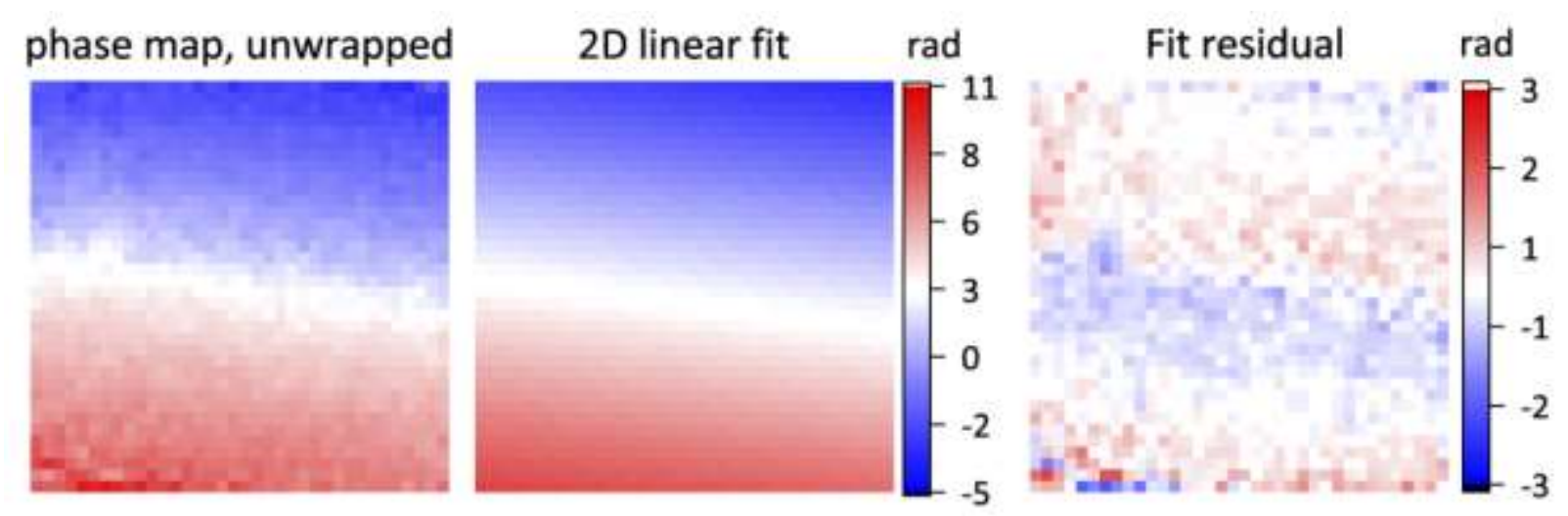

\section{Bandwidth}

- Three-image sequence

- Queue operation

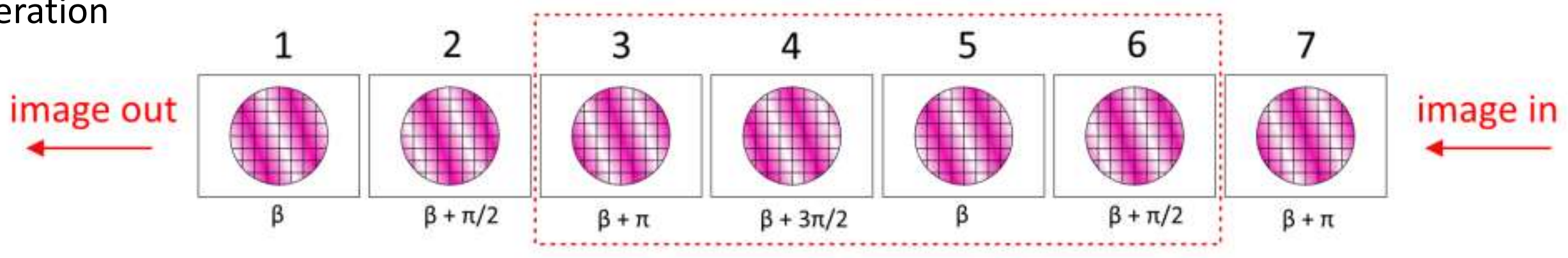




\section{Conclusions:}

1. PSI is simple compared to other atom interferometer techniques.

2. PSI measures two rotation components and one acceleration component at the same time.

3. PSI enables rotation measurement without ambiguity.

4. PSI has a high dynamic range.

5. The SHEEP method returns a phase map with rotation and acceleration information.

6. The SHEEP method does not require a contrast calibration, and it is applicable from the multiple-fringe regime well into the partialfringe regime.
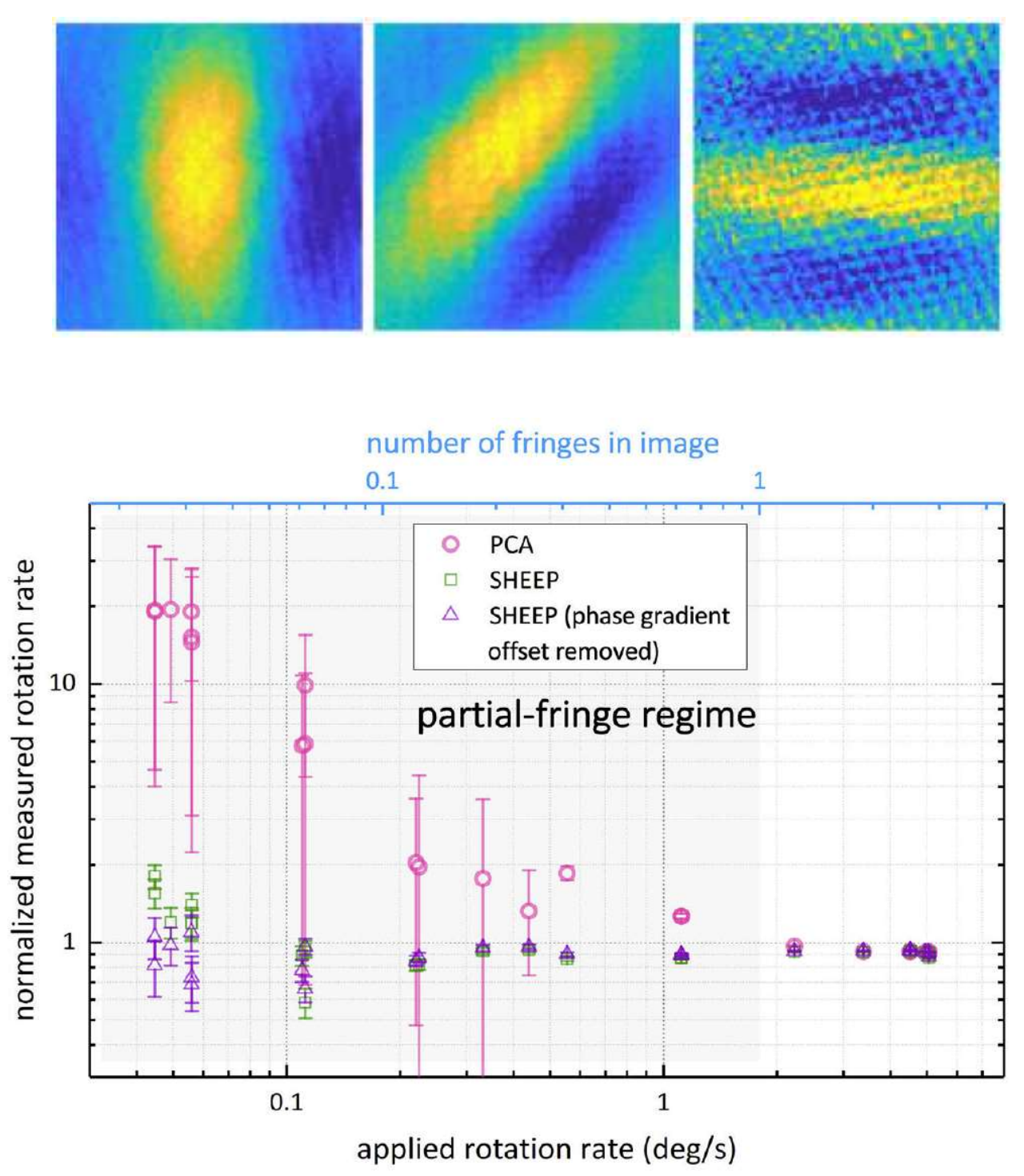